\title{
High-Speed Asymmetric Motion of Thermally Actuated Cilium
}

\author{
Hideyuki Sugioka, ${ }^{*}$ Mako Kubota, and Mitsuhiro Tanaka \\ Department of Mechanical Systems Engineering, \\ Shinshu University 4-17-1 Wakasato, Nagano 380-8553, Japan
}

(Dated: September 24, 2020)

\begin{abstract}
Microorganisms can generate net flows effectively even in a low Reynolds number regime by using asymmetric beating motion of hair-like cilia. Here, we demonstrate a high-speed novel artificial cilium driven by the heat engine using a self-propelled swing motion in a nucleate- to film-boiling regime. In particular, we report the asymmetrical motion of the thermal cilium with an asymmetric joint structure. We believe that our thermal cilium opens a new way to develop a microfluidic circuit.
\end{abstract}

PACS numbers: 47.85.L-s, 47.55.D-, 68.03.-g

\footnotetext{
* hsugioka@shinshu-u.ac.jp
} 


\section{INTRODUCTION}

The pressure-driven flow decreases rapidly as the size of a channel decreases. This problem should be solved to achieve a high integration of microfluidic circuit, such as a Lab-on-a-chip and micro total analysis systems. One clue is cilium or its kinetics because microorganisms can generate net flows effectively even in a low Reynolds number regime by using asymmetric beating motion or metachronal beating motion of hair-like cilia. Therefore, various studies have been devoted for this issue; e.g., Eloy and Lauga numerically reported kinematics of the most efficient cilium [1]. Furthermore, various artificial cilia that mimic biological cilia have been proposed and investigated [2-7]. For example, Evans et al. demonstrated magnetically actuated nanorod arrays in water [3]. Hanasoge et al. demonstrated microfluidic pumping of $\sim 1.35 \mathrm{~mm} / \mathrm{s}$ by using asymmetric motion of magnetic cilia [4]. Toonder reported that electrostatic cilia using curled beams can generate substantial flow of $\sim 0.6 \mathrm{~mm} / \mathrm{s}$ in silicon oil [5]. Masuda et al. reported artificial cilia using a self-oscillating polymer brush surface [6]. Sugioka theoretically demonstrated that artificial cilium using induced charge electroosmosis works in water under AC electric fields [7]. However, artificial cilia using thermal actuator have not been proposed yet to the best of our knowledge. Therefore, in this study, we demonstrate a novel artificial cilium driven by the heat engine [8] using a self-propelled swing motion in a nucleate- to film-boiling regime. In particular, we report the high-speed asymmetrical motion of the thermal cilium with a joint structure.

\section{EXPERIMENTAL METHOD}

Figure 1 shows an experimental setup for the thermally driven cilium having an asymmetric joint structure. As shown in Fig. 1(a), we consider a pair of J-shaped hooks, a micro-heat engine, and the joint structure consisting of three beam elements $\left(m_{0}, m_{1}\right.$, and $\left.m_{2}\right)$ with the joint point $\boldsymbol{X}_{i}(i=0$ to 3$)$, where $L_{m, 0}=\left|\boldsymbol{X}_{0}-\boldsymbol{X}_{1}\right|=8.42 \mathrm{~mm}$, $L_{m, 1}=\left|\boldsymbol{X}_{1}-\boldsymbol{X}_{2}\right|=4.00 \mathrm{~mm}, L_{m, 2}=\left|\boldsymbol{X}_{2}-\boldsymbol{X}_{3}\right|=6.89 \mathrm{~mm}$, and $L=\sum_{i=0}^{2} L_{m, i}=19.31$ mm. Note that $\boldsymbol{X}_{i}=\left(X_{i}, Y_{i}, 0\right)$ is a projection point on $Z=0$ plane. Further, the joint structure was attached to the heat engine and then the heat engine was placed on a pair of J-shaped hooks in water (milli-Q, 18.2 M $\Omega \mathrm{cm}$ ). Figure 1(b) shows the heat engine consisting of a U-shaped nichrome heater and a pair of bent copper wires connected to the heater. Here, 


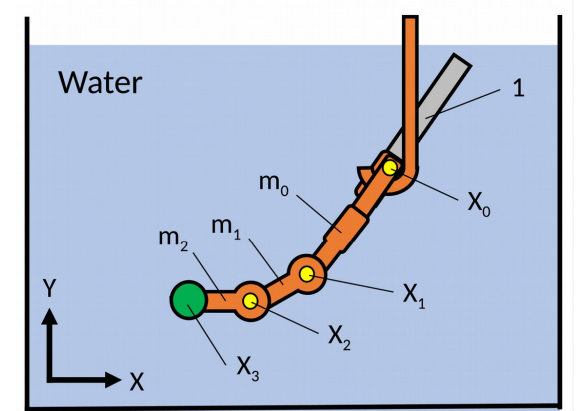

(a)

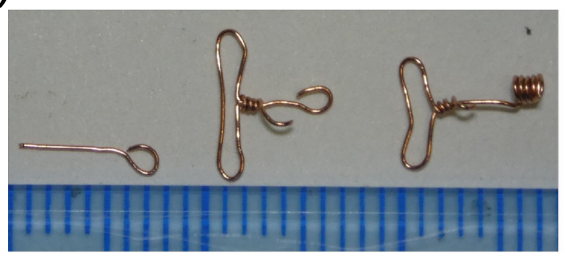

(C)

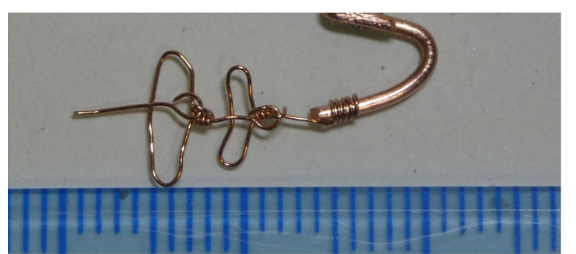

(d) (b)
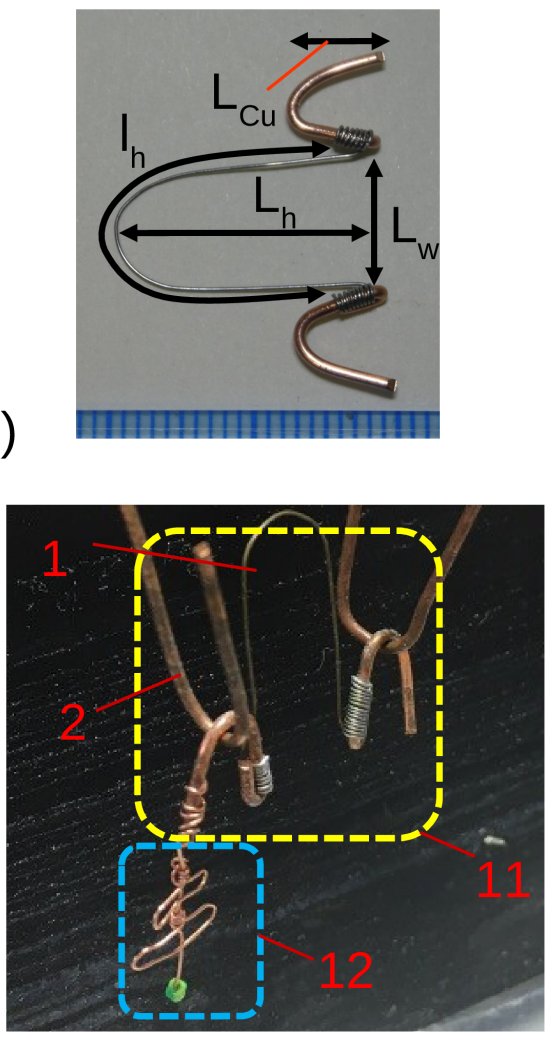

(e)

FIG. 1. (Color online) Experimental setup for the thermally driven cilium having a joint structure.

(a) Experimental Setup. (b) Structure of the micro-heat engine. (c) Three beam elements. (d) Joint structure. (e) Photograph of the entire device. 1: U-shaped nichrome heater. 2: J-shaped hook. 11: micro-heat engine part. 12: joint structure. Here, projection length and diameter of the $\mathrm{Cu}$ wires are $L_{C u}(\simeq 6.5 \mathrm{~mm})$ and $\phi_{C u}(=0.9 \mathrm{~mm})$, respectively, whereas projection length, real length, diameter, and width of the U-shaped heater are $L_{h}(=20 \mathrm{~mm}), l_{h}(=40 \mathrm{~mm}), \phi_{N i C r}(=0.26$ $\mathrm{mm})$, and $L_{w}(=10 \mathrm{~mm})$,respectively.

projection length and diameter of the $\mathrm{Cu}$ wires are $L_{C u}(\simeq 6.5 \mathrm{~mm})$ and $\phi_{C u}(=0.9 \mathrm{~mm})$, respectively, whereas projection length, real length, diameter, width, and surface area of the U-shaped heater are $L_{h}(=20 \mathrm{~mm}), l_{h}(=40 \mathrm{~mm}), \phi_{N i C r}(=0.26 \mathrm{~mm}), L_{w}(=10 \mathrm{~mm})$, and $A\left(=\pi \phi_{N i C r} l_{h}=32.7 \mathrm{~mm}^{2}\right)$, respectively. Note that the heat engine was placed on the J-shaped hooks with the heater up and the $\mathrm{Cu}$ wires down by using the bent structures and it shows a self-propelled swing motion due to the asymmetrical bubble pressure in the nucleate- to film boiling regime [8]. Figure 1(c) shows the three beam elements $\left(m_{0}, m_{1}\right.$, and 
$\left.m_{2}\right)$ made of wires of diameter $\phi_{m}(=0.23 \mathrm{~mm})$, whereas Fig. 1(d) shows the joint structure connected to the one-side of the $\mathrm{Cu}$ wire of the micro-heat engine. As shown in Figs. 1(c) and $1(\mathrm{~d}), m_{0}$ and $m_{1}$ have the deformed oval ring structures that prevent deformation in the $x$ direction. In other words, the oval rings provide asymmetric flexibility with the joint structure; i.e., the joint structure is easy to bent in the $-x$ direction but hard to bend in the $x$ direction [in Fig. 1(a)]. Furthermore, we set this thermal cilium having the joint at rest. Then, upon applying a $\mathrm{DC}$ electric voltage $V_{0}$ to the nichrome heater (with a current $I_{0}$ and a power $P_{0}$ ), we observed a beating motion of this cilium in water under the heat flux $q\left(=\frac{P_{0}}{A}\right)$ of the heater. Subsequently, we determined the position $\boldsymbol{X}_{3}(t)=\left(X_{3}, Y_{3}\right)$ of the tip of the joint structure at time $t$ by using video data of size $640 \times 480$ with a frame rate of $240 \mathrm{fps}$. Such measurements were repeated $N_{f}$ times (typically, $N_{f}=3$ ) under the same conditions and labeled $N=1$ to $N_{f}$ to differentiate them. Moreover, Fig. 1(e) shows the photograph of the entire device. From Fig. 1(e), we can see the relative positions of the pair of J-shaped hooks, joint structure, and heat engine. Note that the entire device was immersed in water and driven by the pressure of the bubbles; i.e., the heat engine part placed in water.

\section{PRINCIPLE OF THE HEAT ENGINE AND ASYMMETRIC MOTION}

Figure 2 shows the principle of the U-shaped heat engine and the asymmetric motion of the joint structure. Although the principle of the heat engine used here is the same as that of the self-propelled swing motion due to the growing instability on heat transfer [8], we briefly explain the principle for reader's convenience. That is, as shown in Fig. 2(a), the Ushaped nichrome wire is initially stopped since $T_{1}=T_{2}\left(>T_{0}\right)$, where $T_{1}$ and $T_{2}$ are the left-

and right-side temperature of the nichrome wire, respectively, and $T_{0}=100{ }^{\circ} \mathrm{C}$ is a boiling temperature of water. However, because the generation of bubbles at $T>T_{0}$ is a stochastic phenomenon, the left average pressure due to the left-side bubbles is slightly different from the right average pressure due to the right-side bubbles and thus the U-shaped wire moves slightly. Once the motion starts, the motion is growing until the driving force balances with the viscous force, because the temperature of the surface in the direction of travel becomes lower than that in the opposite direction and it increases the pressure difference due to the generation of bubbles, as shown in Figs. 2(b) and 2(c). In other words, an initial constant 


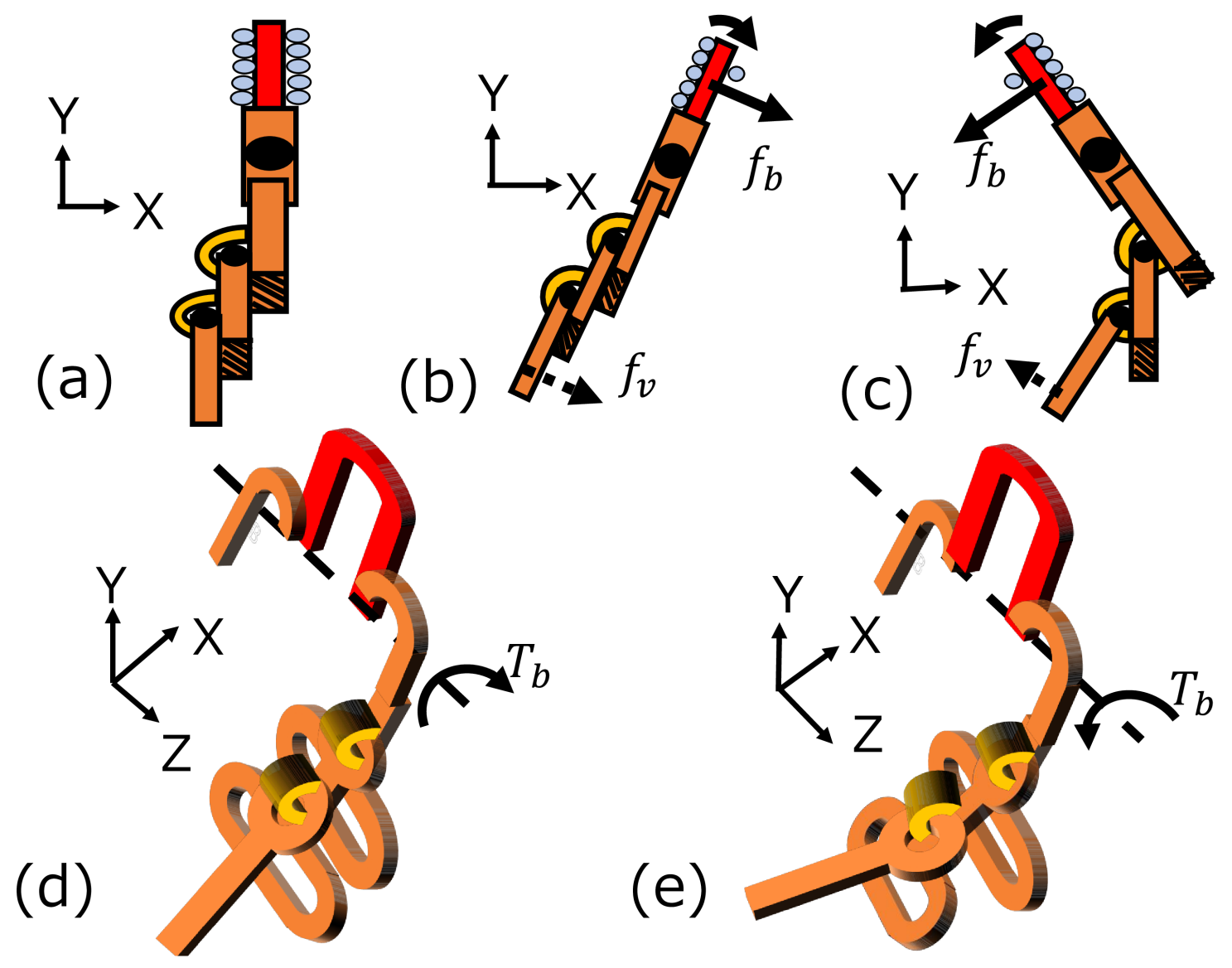

FIG. 2. (Color online) Principle of the heat engine and asymmetric motion. (a) Initial state. (b) Motion in the CCW direction. (c) Motion in the CW direction. (d) Motion in the unbendable $(\mathrm{CCW})$ direction. (e) Motion in the bendable (CW) direction.

heat flux changes into variable once the U-shaped heater moves, because the heat flux at the surface in the direction of travel is different from that in the opposite direction and it depends on the angular velocity. Thus, the motion due to the instability is accelerated and reaches the state of the self-propelled swing motion. Please see the reference [8] for the detail mathematical descriptions. Note that the term "self-propelling" is normally used for a motion induced by an internal energy of a system. Thus, the artificial cilium in the present study is not self-propelling in the narrow sense. However, the energy that cannot be used as it is in the nonequilibrium dissipation system is considered to be a kind of "internal energy". Thus, we expand the concept of "self-propelling", because the motion of our thermal cilium is induced by internal motion due to the fluctuation of the average bubble pressure.

Furthermore, Figs. 2(b) to 2(e) show the principle of asymmetric motion due to the 
joint structure. Here, the planes of the ovals are perpendicular to the $x y$-plane. Thus, on the one hand, the ovals prevent deformation in the $x$ direction, when the U-shaped heater generates force $f_{b}$ and torque $T_{b}$ due bubbles in the counter clockwise $(\mathrm{CCW})$ direction, as shown in Figs. 2(b) and 2(d). On the other hand, as shown in Figs. 2(c) and 2(e), the joint structure deforms when the U-shaped heater generates $f_{b}$ and $T_{b}$ in the clockwise $(\mathrm{CW})$ direction. Note that viscous force $f_{v}$ works to pull the joints elements away from the rings when the U-shaped heater generates $f_{b}$ and $T_{b}$ in the CW direction, while $f_{v}$ works to push the joint elements against the rings when the U-shaped heater generates $f_{b}$ and $T_{b}$ in the CCW direction.

\section{EXPERIMENTAL RESULTS}

Figure 3 shows the photographs of the observed typical asymmetric beating motion of thermally actuated cilium at $t=5.57,5.60,5.65,5.71,5.76$, and $5.78 \mathrm{~s}$ under the condition that $V_{0}=11.6 \mathrm{~V}, I_{0}=9.3 \mathrm{~A}, P_{0}=107.9 \mathrm{~W}, R_{0}=1.25 \Omega$, and $q=3.3 \mathrm{MW} / \mathrm{m}^{2}$. As shown in Figs. 3(a) to 3(c), large deformation of the beam (joint structure) is not observed when the beam moves in the $-x$ direction, whereas the large deformation in the $-x$ direction is observed when the beam moves in the $x$ direction as shown in Figs. 3(d) to 3(f). Thus, the motion in $-x$ direction works as a forward stroke that pushes water strongly, whereas the motion in $x$ direction works as a recovery stroke that pushes water weakly. Here, the planes of the oval rings are perpendicular to the $x y$-plane. Thus, they prevent deformation in the $x$ direction as shown in Figs. 3(a) to 3(c), whereas they do not prevent deformation in the $-x$ direction as shown in Figs. 3(d) and 3(e).

Figure 4(a) depicts the trajectory of $\boldsymbol{X}_{3}$ at $t=0$ to $7.46 \mathrm{~s}$ for $N=1$, which corresponds to the beating motion in Fig. 3. From Fig. 4(a), we find that large asymmetricity exists between the forward and recovery strokes. Furthermore, in Fig. 4(a), the trajectory starts from the origin $(0,0)$, changes from a small loop to large loops, and reaches a steady state, as $t$ increases. From this behavior, we find that a large beating motion results in large asymmetry of outgoing and return routes, which is quantitatively defined as $\left|Y_{3}\left(x_{3}\right)^{U>0}-Y_{3}\left(X_{3}\right)^{U<0}\right|$, where $U \equiv\left|\frac{d \boldsymbol{X}_{3}}{d t}\right|$ is the velocity of the edge of the joint structure. Note that from the overlap of lines around $X_{3} \sim-2.9 \mathrm{~mm}$ shows a large fluctuation of the trajectory at the steady state. Figures 4(b), 4(c), and 4(d) show the dependences of $U, X_{3}$, and $Y_{3}$ on $t$. 


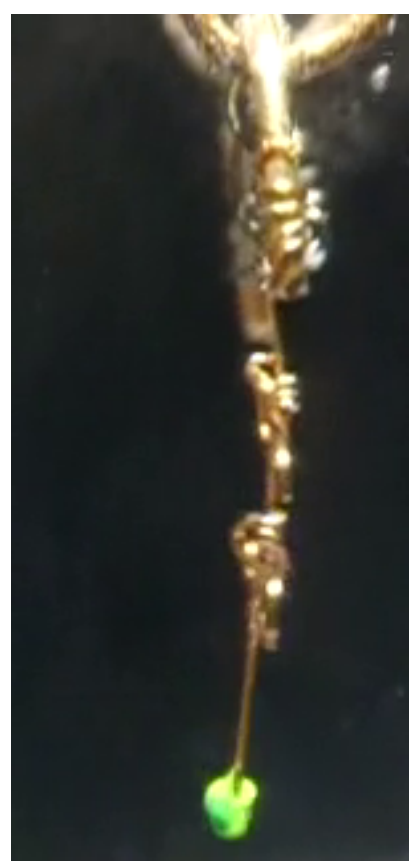

(a) $t=5.57 \mathrm{~s}$

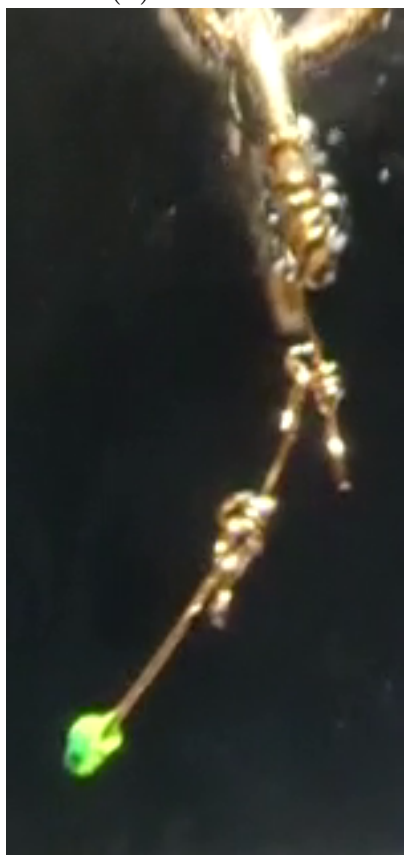

(d) $t=5.71 \mathrm{~s}$

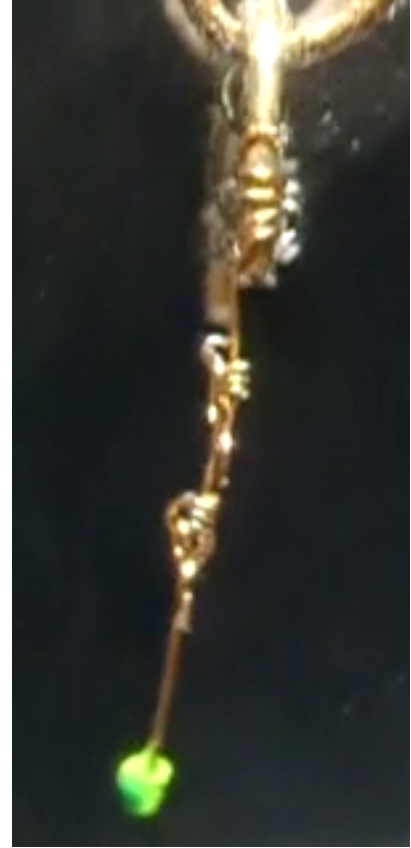

(b) $t=5.60 \mathrm{~s}$

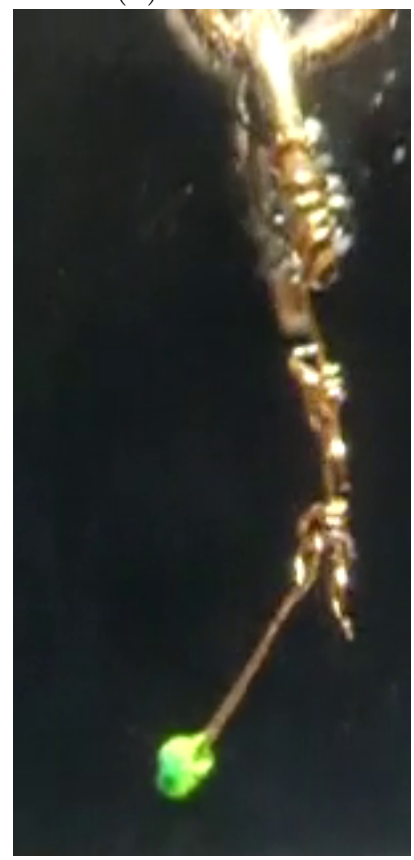

(e) $t=5.76 \mathrm{~s}$

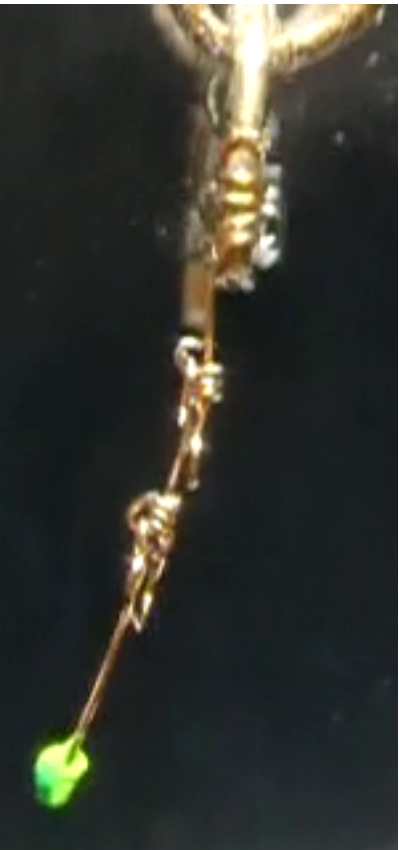

(c) $t=5.65 \mathrm{~s}$

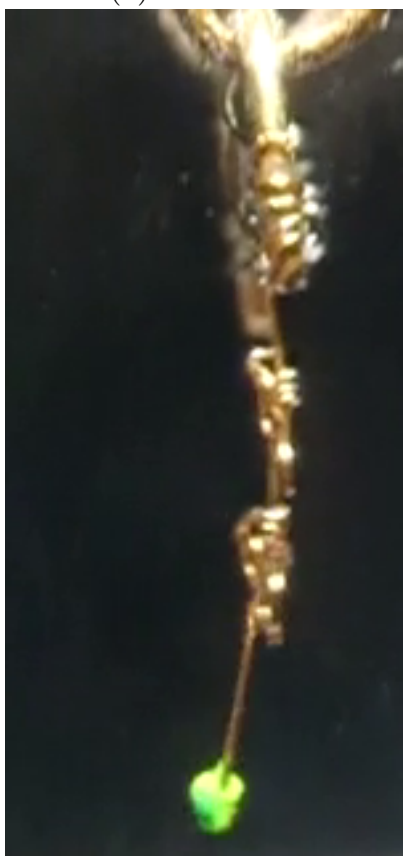

(f) $t=5.78 \mathrm{~s}$

FIG. 3. (Color online) Photographs of the typical asymmetric motion of thermally actuated cilium.

Here, $N=1, V_{0}=11.6 \mathrm{~V}, I_{0}=9.3 \mathrm{~A}, P_{0}=107.9 \mathrm{~W}, R_{0}=1.25 \Omega$, and $q=3.3 \mathrm{MW} / \mathrm{m}^{2} ; \phi_{m}=0.23$ $\mathrm{mm}, L_{m, 0}=\left|\boldsymbol{X}_{0}-\boldsymbol{X}_{1}\right|=8.42 \mathrm{~mm}, L_{m, 1}=\left|\boldsymbol{X}_{1}-\boldsymbol{X}_{2}\right|=4.00 \mathrm{~mm}, L_{m, 2}=\left|\boldsymbol{X}_{2}-\boldsymbol{X}_{3}\right|=6.89 \mathrm{~mm}$, and $L=\sum_{i=0}^{2} L_{m, i}=19.31 \mathrm{~mm}$. 


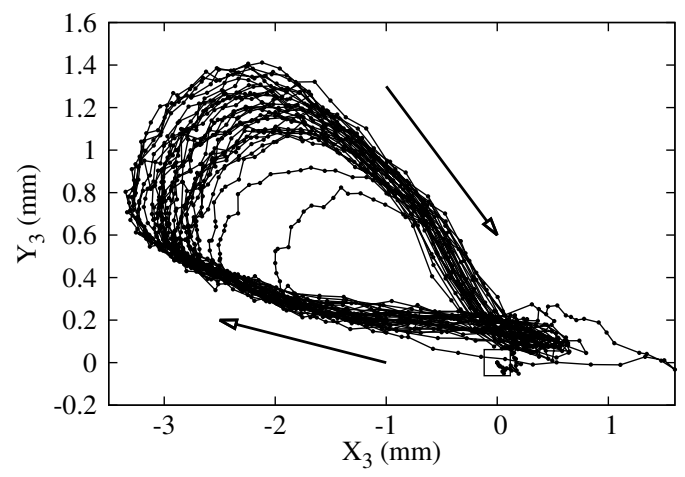

(a) Trajectory $(t=0$ to $7.46 \mathrm{~s})$

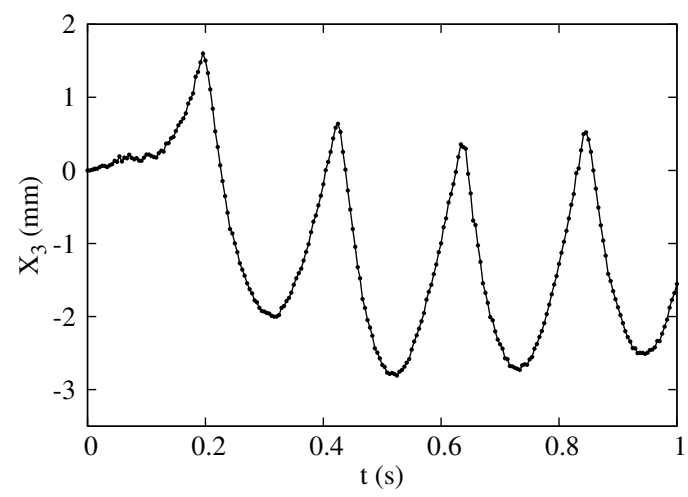

(c) Dependence of $X_{3}$ on $t$

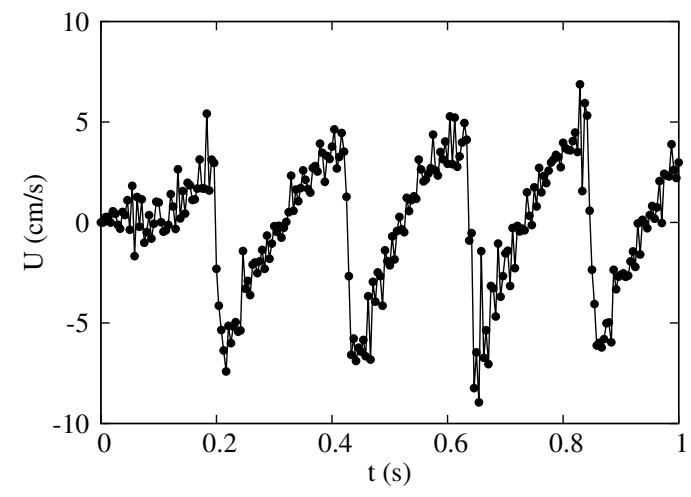

(b) Dependence of $U$ on $t$

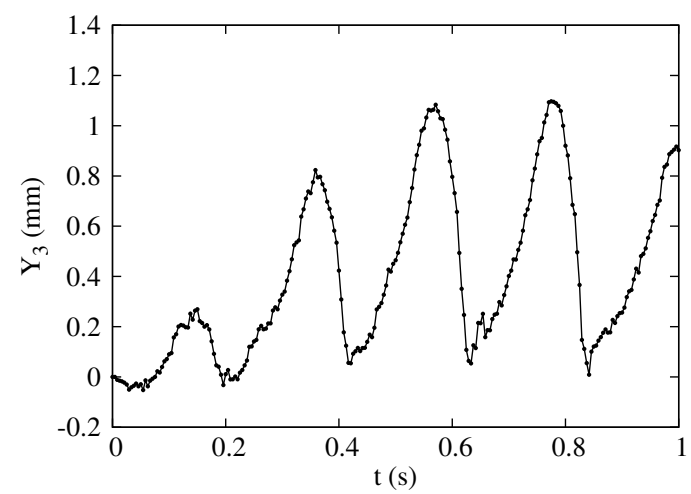

(d) Dependence of $Y_{3}$ on $t$.

FIG. 4. Characteristics of the asymmetric motion of thermally actuated cilium. Here, $N=1$, $V_{0}=11.6 \mathrm{~V}, I_{0}=9.3 \mathrm{~A}, P_{0}=107.9 \mathrm{~W}, R_{0}=1.25 \Omega$, and $q=3.3 \mathrm{MW} / \mathrm{m}^{2}$.

From Fig. 4(b), we find that the maximum velocity of $U$ is approximately $6 \mathrm{~cm} / \mathrm{s}$ in the initial stage $(0.2<t<1)$. Furthermore, from Fig. 4(b) to 4(d), we find that the maximum velocity is obtained at $X_{3} \sim 0$ during the recovery stroke.

Figure 5(a) shows the maximum trajectories at $q=1.9$ to $3.3 \mathrm{MW} / \mathrm{m}^{2}$. Here, the maximum trajectory is defined as the trajectory of one cycle when $\Delta Y_{a} \equiv Y_{3}^{\max }-Y_{3}^{\min }$ is maximum at each heat flux, where $Y_{3}^{\max }$ and $Y_{3}^{\min }$ are the maximum and minimum values of $Y_{3}$ at the each heat flux. From Fig. 5(a), we find that large trajectories are observed at $q \geq 2.3 \mathrm{MW} / \mathrm{m}^{2}$ and $\Delta Y_{a}$ shows the asymmetricity of the beating motion of the cilium. Figures $5(\mathrm{~b}), 5(\mathrm{c})$, and $5(\mathrm{~d})$ show the dependences of $\Delta Y_{a}, U_{\max }$, and $f_{b}$, respectively on $q$, where $U_{\max }$ is the maximum value of $U$ and $f_{b}$ is the frequency of the beating motion. As shown in Fig. 5(b), $\Delta Y_{a}$ increases monotonically at $q>2 \mathrm{~W} / \mathrm{m}^{2}$ as $q$ increases, whereas $U_{\max } \sim 12 \mathrm{~cm} / \mathrm{s}$ at $q \geq 2.8 \mathrm{~W} / \mathrm{m}^{2}$ as shown in Fig. 5(c). Furthermore, as shown in Fig. 5(d), $f_{b} \sim 4.5 \mathrm{~Hz}$ when we observe a significant beating motion. 


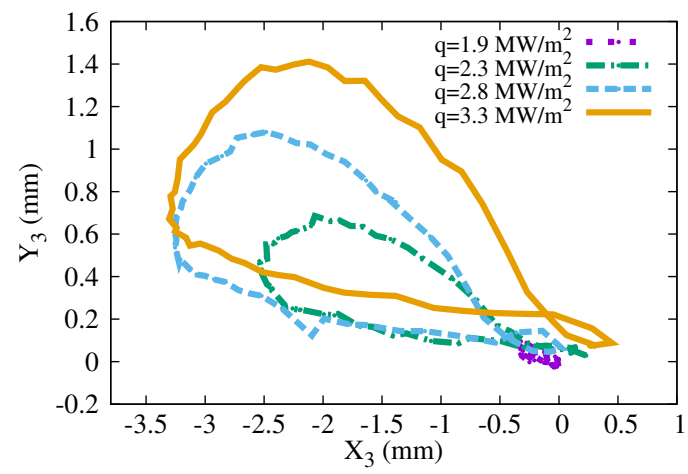

(a) Trajectories at $q=1.9$ to $3.3 \mathrm{MW} / \mathrm{m}^{2}$

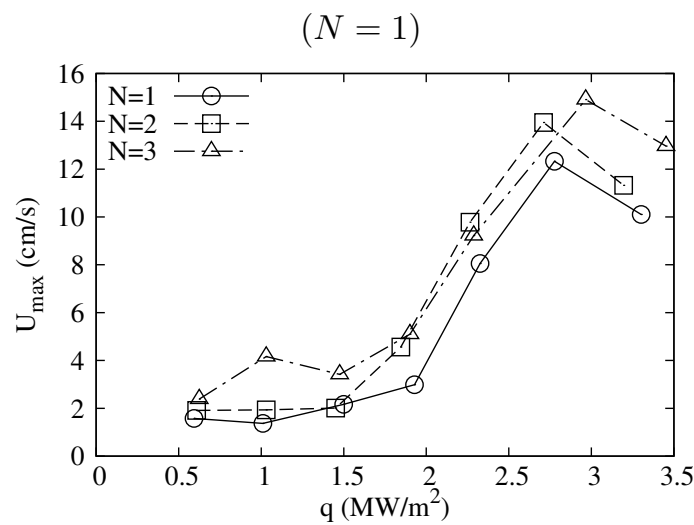

(c) Dependence of $U_{\max }$ on $q$.

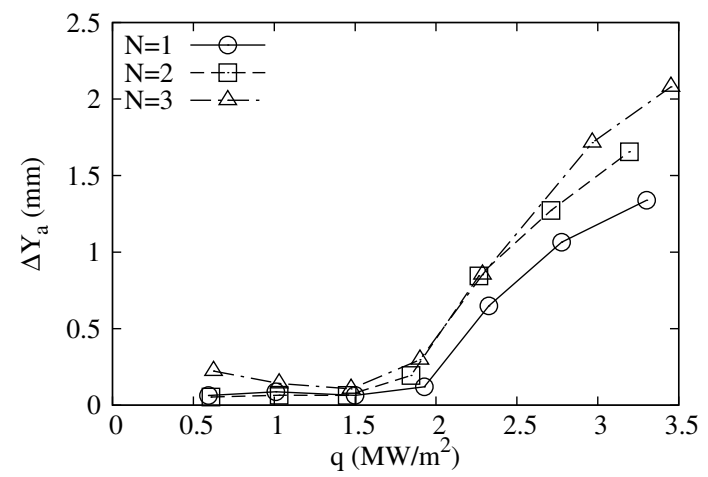

(b) Dependence of $\Delta Y_{a}=Y_{3}^{\max }-Y_{3}^{\min }$ on $q$.

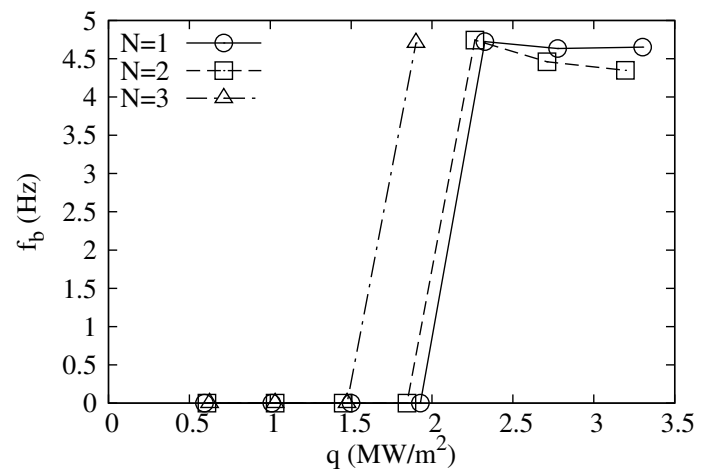

(d) Dependence of $f_{p}$ on $q$.

FIG. 5. (Color online) Dependences on $q$ for $L=19.31 \mathrm{~mm}$. Here, $N_{f}=3, L_{m, 0}=\left|\boldsymbol{X}_{0}-\boldsymbol{X}_{1}\right|=8.42$ $\mathrm{mm}, L_{m, 1}=\left|\boldsymbol{X}_{1}-\boldsymbol{X}_{2}\right|=4.00 \mathrm{~mm}$, and $L_{m, 2}=\left|\boldsymbol{X}_{2}-\boldsymbol{X}_{3}\right|=6.89 \mathrm{~mm}$.

\section{DISCUSSION}

\section{A. The threshold and miniaturization of the simple pendulum having a thin film}

\section{heater}

Although we use a U-shaped heater to demonstrate our concept, it can be replaced by a thin film heater. Thus, to miniaturize our device, we consider the pendulum having a thin film heater on the surface, as shown in Fig. 6(a). Here, the pendulum is placed in water of density $\rho_{w} \simeq 1000 \mathrm{~kg} / \mathrm{m}^{3}$ and the length, width, thickness, volume, and density are $l, w$, $d, V=l w d$, and $\rho_{p}\left(2330 \mathrm{Kg} / \mathrm{m}^{3}\right.$ for $\left.\mathrm{Si}\right)$, respectively. In this case, similar to the previous 


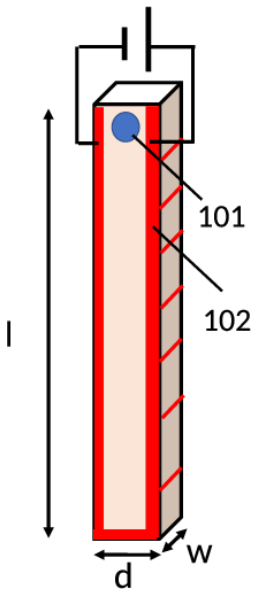

(a) Pendulum

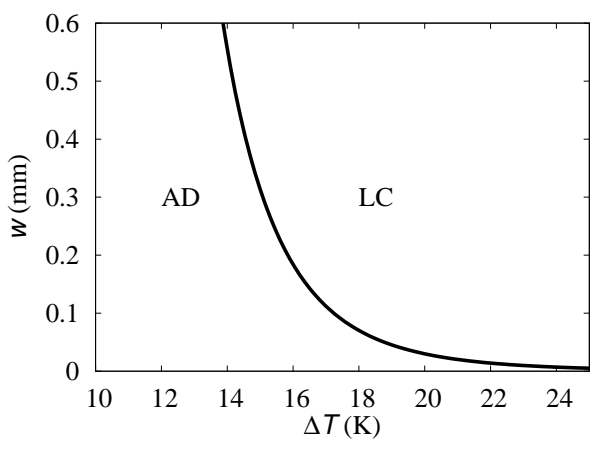

(b) Two parameter ( $w$ vs. $\Delta T)$ phase diagram

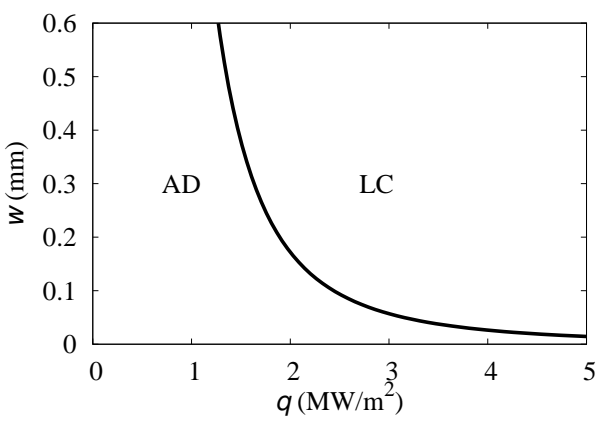

(c) Two parameter ( $w$ vs. $q$ ) phase diagram

FIG. 6. (Color online) The threshold of the simple pendulum having a thin film heater.

study [8], the government equations of the pendulum are described as follows:

$$
\begin{array}{r}
I \ddot{\theta} \simeq-\frac{m^{\prime} g l}{2} \sin \theta+T_{b}-T_{v}, \\
T_{v} \simeq 12 \mu l\left(\frac{l}{2}\right)^{2} \dot{\theta}=3 \mu l^{3} \dot{\theta}, \\
T_{b} \simeq \frac{l^{2} w}{2} \Delta P R_{a} R_{b}, \\
\Delta P=P_{s}\left(T_{0}+\Delta T\right)-P_{s}\left(T_{0}\right), \\
P_{s} \simeq p_{a} \mathrm{e}^{-\frac{r_{0}}{R T}}, \\
R_{a}=\left(1-\mathrm{e}^{-\frac{l|\dot{\theta}|}{2 u_{c}}}\right) \frac{\dot{\theta}}{|\dot{\theta}|}, \\
R_{b}=\left(1-\mathrm{e}^{\frac{-\Delta T}{\Delta T_{c}}}\right)^{8},
\end{array}
$$

where $I=\frac{m^{\prime} l^{2}}{3}$ is the moment of inertia, $m^{\prime}=\left(\rho_{p}-\rho_{w}\right) V$ is an effective mass, $T_{b}$ and $T_{v}$ are the torques due to bubbles and viscosity, respectively, $\Delta T$ is an average surface temperature difference of the heater (which is measured from $T_{0}=100{ }^{\circ} \mathrm{C}$ ), $\Delta P$ is an intrinsic pressure difference, $P_{s}$ is a saturated vapor pressure determined by the Clausius-Clapeyron equation [with the constant $p_{a}=0.1013 \mathrm{MPa}$, the evaporation latent heat $r_{0}=2256.9 \mathrm{~kJ} / \mathrm{kg}$, and the gas constant $R=0.4616 \mathrm{~kJ} /(\mathrm{kg} \mathrm{K})$ of water], $R_{a}$ is the availability rate of the force difference due to the broken symmetry of the right and left temperature resulting from the non-zero value of $\dot{\theta}, R_{b}$ is an effective surface bubble coverage rate, $u_{c} \simeq 5 \mathrm{~mm} / \mathrm{s}$ is a critical velocity causing a significant broken symmetry, $\Delta T_{c} \simeq 60 \mathrm{~K}$ is the Leidenfrost temperature, 
and $\theta$ is an angle of the pendulum measured from the vertical direction. By transforming Eq. (1), we obtain

$$
\begin{array}{r}
\dot{\theta}=\omega_{p}, \\
\dot{\omega}=-C \sin \theta+f_{p}(\theta, \dot{\theta}), \\
C=\frac{3 g}{2 l}, \\
f(\theta, \dot{\theta}) \simeq \frac{\frac{l^{2} w}{2} \Delta P R_{a} R_{b}-3 \mu l^{3} \dot{\theta}}{\frac{1}{3} m^{\prime} l^{2}}=\frac{w \Delta P R_{a} R_{b}-18 \mu l \dot{\theta}}{2 m^{\prime}} .
\end{array}
$$

Here, since $R_{a} \sim \frac{l}{2 u_{c}} \dot{\theta}$ at $l|\dot{\theta}| \ll 2 u_{c}$, we obtain $w \Delta P R_{a} R_{b}-18 \mu l \dot{\theta} \sim\left(w \Delta P R_{b} \frac{l}{2 u_{c}}-18 \mu l\right) \dot{\theta}$ for a small motion of the pendulum. Thus, the condition for the amplified oscillation to start is written as

$$
w \geq \frac{36 \mu u_{c}}{\Delta P R_{b}}
$$

Therefore, we find that we can miniaturize our device under the condition of Eq. (12); i.e., our device can be miniaturized regardless of the length. Furthermore, Eq. (12) provides the threshold from a dynamical system point of view. That is, Figs 6(b) and 6(c) show the two phase diagrams denoting amplitude death (AD) state to limiting cycle (LC) state for $w$ vs. $\Delta T$ and for $w$ vs. $q$, respectively. Here, similar to the previous study [8], we use the formulation that $q=\left(\frac{\Delta T}{\Delta T_{A}}\right)^{3} q_{A}$, where $\Delta T_{A}=20 \mathrm{~K}$ and $q_{A}=3.81 \mathrm{MW} / \mathrm{m}^{2}$. From Figs $6(\mathrm{~b})$ and 6(c), we find that the threshold width $w_{0}=\frac{36 \mu u_{c}}{\Delta P R_{b}}$ is approximately $50 \mu \mathrm{m}$ at $\Delta T_{A} \simeq 20$ $\mathrm{K}$ and $q_{A} \simeq 4 \mathrm{MW} / \mathrm{m}^{2}$. 
B. Characteristics of the miniaturized simple pendulum using the self-propelled micro heat engine

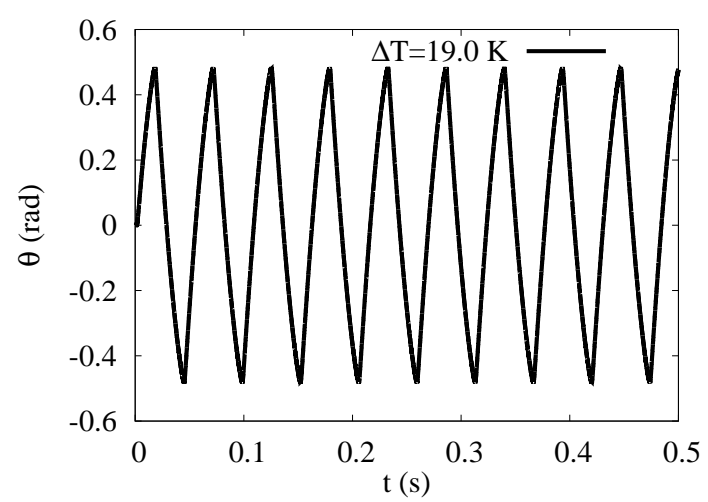

(a) Dependence of $\theta$ on $t$

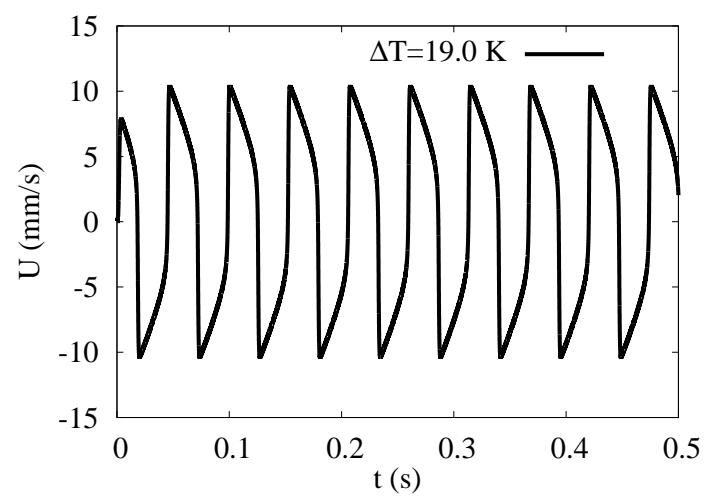

(c) Dependence of $U$ on $t$

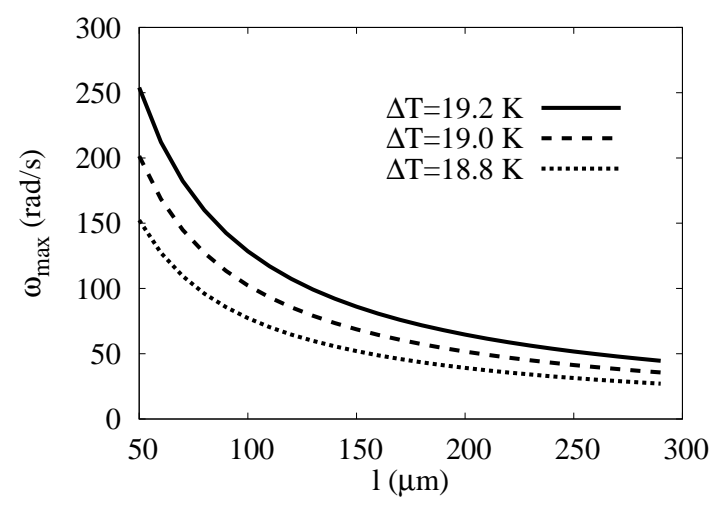

(e) Dependence of $\omega_{\max }$ on $l$

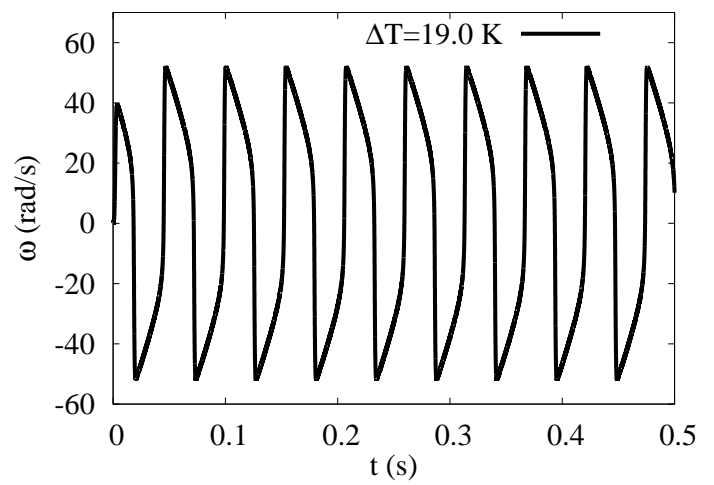

(b) Dependences of $\omega$ on $t$

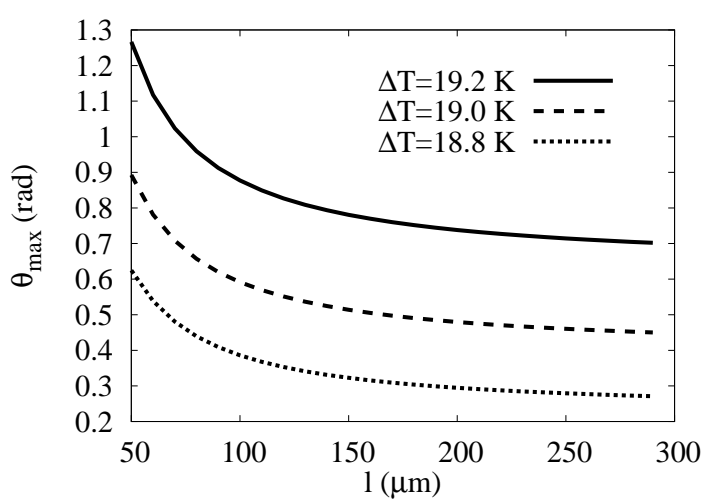

(d) Dependences of $\theta_{\max }$ on $l$

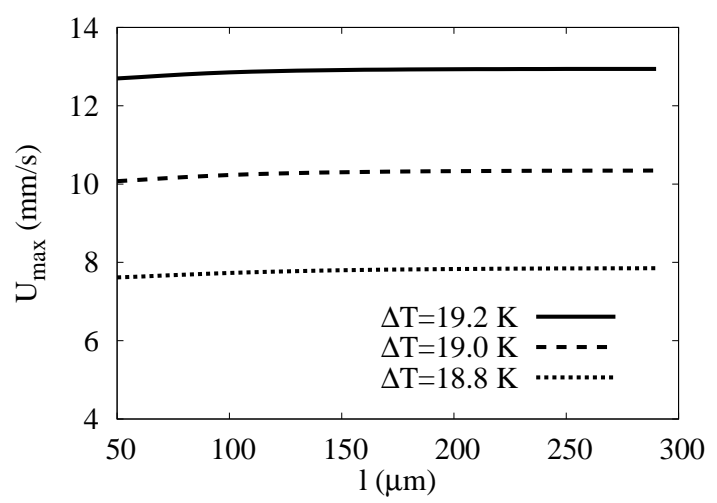

(f) Dependences of $U_{\max }$ on $l$

FIG. 7. Characteristics of the miniaturized simple pendulum using the self-propelled micro heat engine. Here, we assume that $m^{\prime}=0.266 \mu \mathrm{g}, w=100 \mu \mathrm{m}$, and $d=10 \mu \mathrm{m}$. 
Figure 7 shows the characteristics of the miniaturized simple pendulum using the selfpropelled micro heat engine under the condition that $m^{\prime}=0.266 \mu \mathrm{g}, w=100 \mu \mathrm{m}$, and $d=10 \mu \mathrm{m}$. Specifically, Figs $7(\mathrm{a}), 7(\mathrm{~b})$, and 7(c) show the dependences of $\theta$, $\omega$, and $U(=\omega l)$ on $t$, respectively, at $l=200 \mu \mathrm{m}$ and $\Delta T=19.0 \mathrm{~K}\left(q=3.27 \mathrm{MW} / \mathrm{m}^{2}\right)$. As shown in those figures, we find that $\theta_{\max }=0.48 \mathrm{rad}, \omega_{\max }=51,6 \mathrm{rad} / \mathrm{s}, U_{\max }=10.3 \mathrm{~mm} / \mathrm{s}$, and $f_{0}=43.1 \mathrm{~Hz}$; i.e., the pendulum have the enough performance even for a small length. Furthermore, we find that the motion of the pendulum becomes steady within two cycles (within $\sim 0.01$ s); i.e., the response time of the miniaturized device is very short. Moreover, we find that the wave form of $\omega(t)[U(t)]$ is different from the sinusoidal wave. That is, $\omega$ increases rapidly but linearly up to the lowest point from $\omega=0$ at $\theta=-\theta_{\max }$. After passing the lowest point, $\omega$ decreases gradually and becomes zero at $\theta=\theta_{\max }$. Similarly, $\omega$ decreases rapidly but linearly up to the lowest point from $\omega=0$ at $\theta=\theta_{\text {max }}$. After passing the lowest point, $\omega$ increases gradually and becomes zero at $\theta=\theta_{\max }$. As a result, $\theta$ changes linearly in the direction toward the lowest point, while $\theta$ changes gradually in the direction to go up. This is because the effect of gravity is added to the propulsion force due to the bubbles when the pendulum goes down, whereas the effect of gravity is subtracted from the propulsion force. Furthermore, Figures $7(\mathrm{~d}), 7(\mathrm{e})$, and $7(\mathrm{f})$ show the dependences of $\theta_{\max }$, $\omega_{\max }$, and $U_{\max }$ on $l$, respectively, at $\Delta T=18.8,19.0$, and $19.2 \mathrm{~K}(q=3.16,3.27$, and 3.37 MW/ $/ \mathrm{m}^{2}$ ). As shown in Figs. $7(\mathrm{~d})$ and $7(\mathrm{e}), \theta_{\max }$ and $\omega_{\max }$ increase as $l$ becomes small; i.e., the performance becomes large for the miniaturization of our device. Furthermore, as shown in Fig. $7(\mathrm{f}), U_{\max }\left(=l \omega_{\max }\right)$ becomes almost constant because $\omega_{\max }$ increases rapidly as $l$ decreases; the performance of the velocity in a macro scale is maintained. Hence, the performance of our device increases due to the miniaturization. 


\section{Theory of a double pendulum using the self-propelled micro heat engine}

The government equations of a double pendulum consisting of two beam structures (Beams 1 and 2) of length $l_{1}$ and $l_{2}$ and of mass $m_{1}$ and $m_{2}$ in water are described as

$$
\begin{array}{r}
I_{1} \ddot{\theta}_{1} \simeq-\frac{1}{2} m_{1} l_{1} g \sin \theta_{1}+\frac{1}{2} l_{1} f_{1}+l_{1} \boldsymbol{T}_{1} \cdot \boldsymbol{q}\left(\theta_{1}\right), \\
I_{2} \ddot{\theta}_{2} \simeq \frac{1}{2} l_{2}\left(-\boldsymbol{T}_{1}\right) \cdot\left(-\boldsymbol{q}\left(\theta_{2}\right)\right), \\
m_{2} \ddot{\boldsymbol{r}}_{2}=-m_{2} g \boldsymbol{j}-\boldsymbol{T}_{1}+f_{2} \boldsymbol{q}\left(\theta_{2}\right), \\
f_{1}=w l_{1} \Delta P R_{a} R_{b}-12 \mu l_{1}\left(\frac{l_{1}}{2} \dot{\theta}_{1}\right) \\
f_{2}=-12 \mu l_{2} \dot{\boldsymbol{r}}_{2} \cdot \boldsymbol{q}\left(\theta_{2}\right) \\
\boldsymbol{r}_{2}=l_{1} \boldsymbol{p}\left(\theta_{1}\right)+\frac{1}{2} l_{2} \boldsymbol{p}\left(\theta_{2}\right) \\
\boldsymbol{p}(\theta)=\sin \theta \boldsymbol{i}-\cos \theta \boldsymbol{j} \\
\boldsymbol{q}(\theta)=\cos \theta \boldsymbol{i}+\sin \theta \boldsymbol{j},
\end{array}
$$

where $r_{2}$ is the center of gravity of Beam 2, $\boldsymbol{T}_{1}$ is the force that Beam 1 receives from Beam2 at the junction, $-\boldsymbol{T}_{1}$ is the force that Beam 2 receives from Beam1 at the junction, $I_{1}=$ $\int_{0}^{l_{1}} x^{2} \frac{m_{1}}{l_{1}}=\frac{1}{3} m_{1} l_{1}^{2}$ is a moment of inertia around the fixed point (origin), $I_{2}=\int_{-0.5 l_{2}}^{0.5 l_{2}} x^{2} \frac{m_{2}}{l_{2}}=$ $\frac{1}{12} m_{2} l_{2}^{2}$ is a moment of inertia around the center of gravity of Beam $2, f_{1}$ is the force due to bubbles and viscosity for Beam1, and $f_{2}$ is the viscous force for Beam2. Here, we assume that a self-propelled micro heat engine (thin film heater) is equipped only on Beam 1.

From Eq. (15), we obtain

$$
\boldsymbol{T}_{1}=-m_{2}\left(\ddot{\boldsymbol{r}_{2}}+g \boldsymbol{j}\right)+f_{2} \boldsymbol{q}\left(\theta_{2}\right)
$$

Thus, we can calculate time evolution of $\theta_{1}$ and $\theta_{2}$ from Eqs. (13) and (14). In detail, by transforming Eqs. (13) and (14), we obtain

$$
\begin{aligned}
& \dot{\theta_{1}}=\omega_{1}, \\
& \dot{\theta_{2}}=\omega_{2} \text {, } \\
& \dot{\omega}_{1}=\frac{1}{I_{1}}\left[-\frac{1}{2} m_{1} l_{1} g \sin \theta_{1}+\frac{1}{2} l_{1} f_{1}+l_{1} \boldsymbol{T}_{1} \cdot \boldsymbol{q}\left(\theta_{1}\right)\right], \\
& \dot{\omega}_{2}=\frac{1}{I_{2}} \frac{1}{2} l_{2} \boldsymbol{T}_{1} \cdot \boldsymbol{q}\left(\theta_{2}\right) \text {. }
\end{aligned}
$$

By considering that $\ddot{r}_{2}=l_{1} \ddot{\theta}_{1}\left(\boldsymbol{q}\left(\theta_{1}\right)-\dot{\theta}_{1}^{2} \boldsymbol{p}\left(\theta_{1}\right)\right)+\frac{1}{2} l_{2} \ddot{\theta}_{2}\left(\boldsymbol{q}\left(\theta_{2}\right)-\dot{\theta}_{2}^{2} \boldsymbol{p}\left(\theta_{2}\right)\right), \boldsymbol{T}_{1} \cdot \boldsymbol{q}\left(\theta_{1}\right)=-m_{2}\left(\ddot{r}_{2}\right.$. $\left.\boldsymbol{q}\left(\theta_{1}\right)+g \boldsymbol{q}\left(\theta_{1}\right) \cdot \boldsymbol{j}\right)+f_{2} \boldsymbol{q}\left(\theta_{1}\right) \cdot \boldsymbol{q}\left(\theta_{2}\right)$, and $\boldsymbol{T}_{1} \cdot \boldsymbol{q}\left(\theta_{2}\right)=-m_{2}\left(\ddot{r}_{2} \cdot \boldsymbol{q}\left(\theta_{2}\right)+g \boldsymbol{q}\left(\theta_{2}\right) \cdot \boldsymbol{j}\right)+f_{2}$, we 
obtain

$$
\frac{d}{d t} \boldsymbol{\theta}=\boldsymbol{\omega}
$$

$$
\frac{d}{d t} \boldsymbol{A} \boldsymbol{\omega}=\boldsymbol{\varphi}
$$

where

$$
\begin{gathered}
\boldsymbol{A}=\left(\begin{array}{cc}
a_{11} & a_{12} \\
a_{21} & a_{22}
\end{array}\right)=\left(\begin{array}{cc}
I_{1}+\frac{1}{4} m_{2} l_{1}^{2} & \frac{1}{4} m_{2} l_{1} l_{2} \cos \left(\theta_{2}-\theta_{1}\right) \\
\frac{1}{2} m_{2} l_{1} l_{2} \cos \left(\theta_{2}-\theta_{1}\right) & I_{2}+\frac{1}{4} m_{2} l_{2}^{2} a
\end{array}\right) \\
\boldsymbol{\varphi}=\left[\begin{array}{c}
\varphi_{1} \\
\varphi_{2}
\end{array}\right]=\left[\begin{array}{c}
-\frac{1}{2}\left(m_{1}+m_{2}\right) g l_{1} \sin \theta_{1}+\frac{1}{4} m_{2} l_{1} l_{2} \dot{\theta}_{2}^{2} \sin \left(\theta_{2}-\theta_{1}\right)+\frac{1}{2} l_{1}\left[f_{1}+f_{2} \cos \left(\theta_{2}-\theta_{1}\right)\right] \\
-\frac{1}{2} m_{2} g l_{2} \sin \theta_{2}-\frac{1}{2} m_{2} l_{1} l_{2} \dot{\theta}_{1}^{2} \sin \left(\theta_{2}-\theta_{1}\right)+\frac{1}{2} l_{1} f_{2}
\end{array}\right] \\
\boldsymbol{\omega}=\left[\begin{array}{c}
\omega_{1} \\
\omega_{2}
\end{array}\right]
\end{gathered}
$$

and

$$
\boldsymbol{\theta}=\left[\begin{array}{l}
\theta_{1} \\
\theta_{2}
\end{array}\right]
$$

Therefore, by solving $\dot{\boldsymbol{\omega}}=\boldsymbol{A}^{-1} \boldsymbol{\varphi}$ and $\dot{\boldsymbol{\theta}}=\boldsymbol{\omega}$ numerically, we can determine the time evolution of $\theta_{1}, \theta_{2}, \omega_{1}$, and $\omega_{2}$. 
D. The trajectories and frequencies of various pendulums using the self-propelled micro heat engine

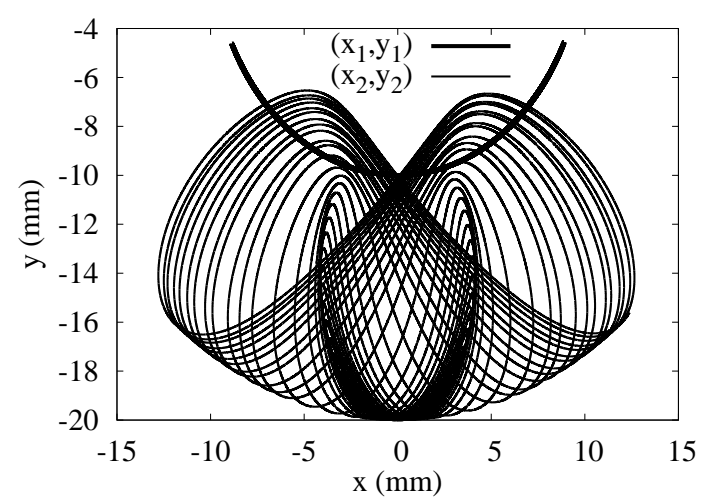

(a) Trajectory of the SDP

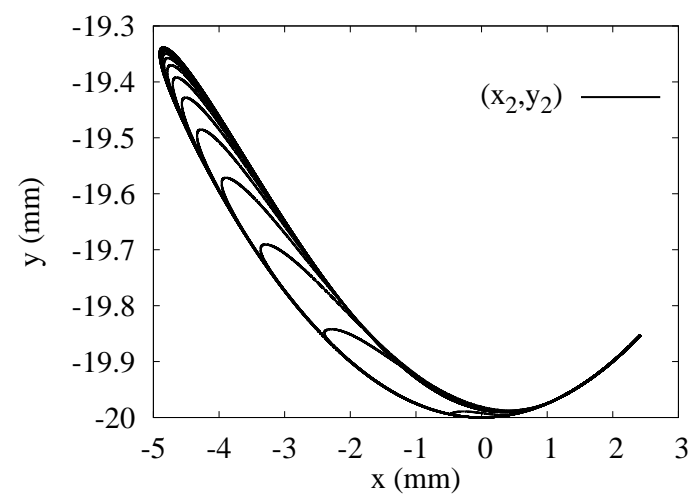

(c) Trajectory of the ASDP

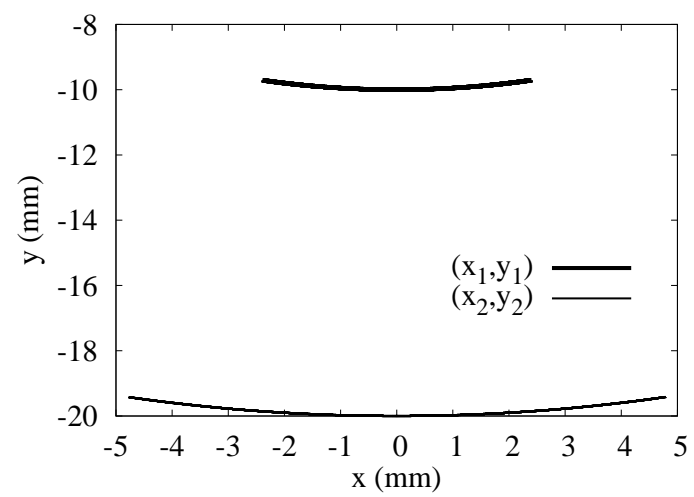

(e) Trajectory of the SP

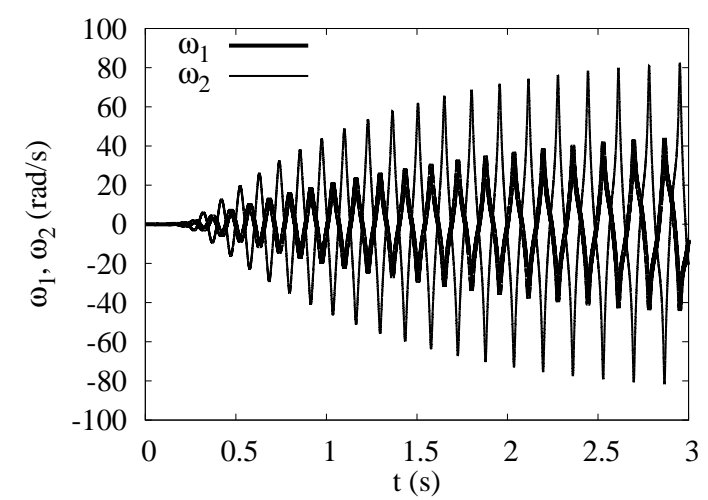

(b) Dependences of $\omega_{1}$ and $\omega_{2}$ on $t$ for the SDP

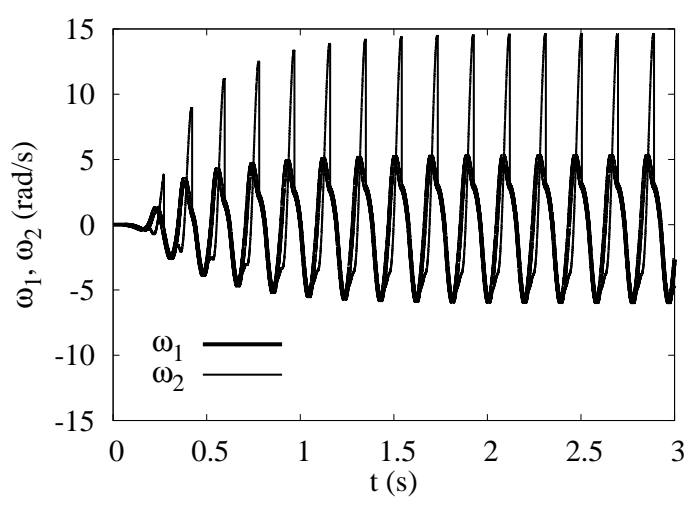

(d) Dependences of $\omega_{1}$ and $\omega_{2}$ on $t$ for the ASDP

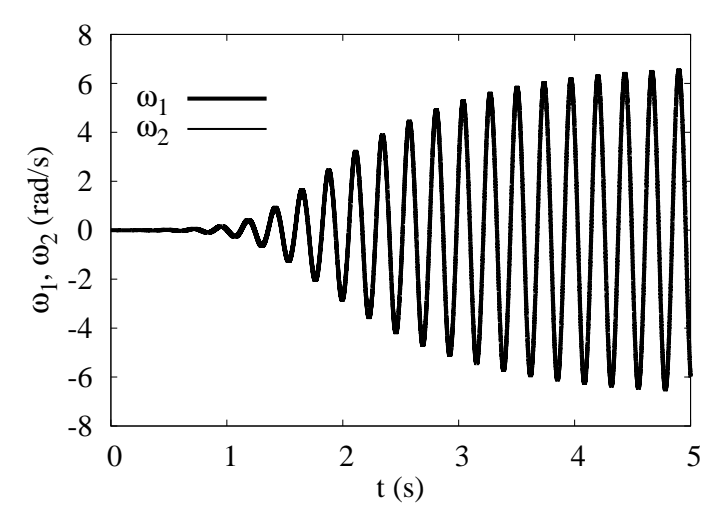

(f) Dependences of $\omega_{1}$ and $\omega_{2}$ on $t$ for the SP

FIG. 8. Comparison of of various pendulums using the self-propelled micro heat engine. Here, we assume that $\Delta T=19.5 \mathrm{~K}, d=2 \mathrm{~mm}, w=2 \mathrm{~mm}, l_{1}=l_{2}=10.0 \mathrm{~mm}$, and $m_{1}=m_{2}=53.2 \mathrm{mg}$. 
Figure 8 shows the comparison of various pendulums using the self-propelled micro heat engine under the conditions that $\Delta T=19.5 \mathrm{~K}, l_{1}=l_{2}=10.0 \mathrm{~mm}$, and $m_{1}=m_{2}=53.2 \mathrm{mg}$, on the basis of the formulations of Secs. V.A and V.C. Specifically, Figs. 8(a), 8(c), and 8(e) show the trajectories for a symmetrical double pendulum (SDP), an asymmetrical double pendulum (ASDP), and a single pendulum (SP), respectively, whereas Figs. 8(b), 8(d), and 8(f) show the dependences of $\omega_{1}$ and $\omega_{2}$ on $t$ for the SDP, ASDP, and SP, respectively. In Fig. 8, $\left(x_{1}, y_{1}\right)$ and $\left(x_{2}, y_{2}\right)$ show the edge positions of Beams 1 and 2, respectively. Furthermore, the calculation results of the ASDP are obtained by imposing the condition that $\theta_{2}=\theta_{1}$ when $\theta_{2}$ becomes larger than $\theta_{1}$ in the numerical simulations. As shown in Figs. 8(a), 8(c), and 8(e), we find that the amplitude of $\left(x_{2}, y_{2}\right)$ decreases in the order the SP, SDP, ASDP. In other words, the performance the efficiency of motion decreases in the order the SP, SDP, ASDP. This is because the viscous forces are in the order the SP, SDP, ASDP. In particular, the average viscous force of the ASDP is much smaller than others because of the bending in the right and left directions. Furthermore, the left-side amplitude of the ASDP is the same as that of the SP, whereas the right-side amplitude of the ASDP is much smaller than that of the SP, since the driving force of Beam 1 is not effectively transmitted to Beam 2 during the motion of Beam 1 in the left direction because of the bending of the double pendulum. Moreover, as shown in Figs. 8(b), 8(d), and 8(f), we find that the frequencies of $\omega_{2}$ for the SP, ASDP, and SDP are 4.31, 5.17, and $5.86 \mathrm{~Hz}$. This is reasonable because the theoretical natural frequency $f_{0}^{s p}$ for the SP is described as $f_{0}^{s p}=\frac{1}{2 \pi} \sqrt{\frac{3 g}{2 l}}=4.31$ $\mathrm{Hz}$, whereas the natural frequencies of the others become higher than that since the effective length of the pendulum becomes smaller because of the bending of the beams for the ASDP and the SDP.

\section{E. The efficiency for the ASDP}

Figure 9 shows the efficiency for the ASDP in Sec. V.C. Here, a potential net pumping volume flow rate $Q_{p}$ is defined as

$$
Q_{p} \equiv S w f_{b}
$$

Here, originally, $Q_{p}$ is a net volume swept by a cilium per unit time, since the net flow of the region swept in the outgoing and return routes is zero because of the scallop theorem, which states that time-symmetric motion cannot achieve net displacement in a low Reynolds 


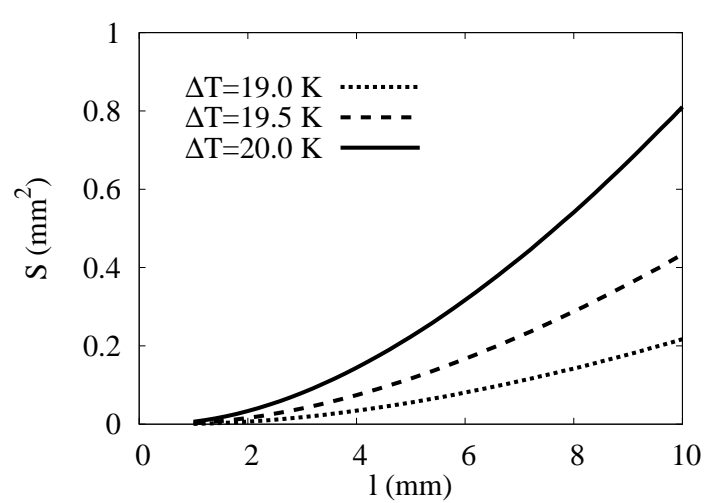

(a) Dependence of $S$ on $l$

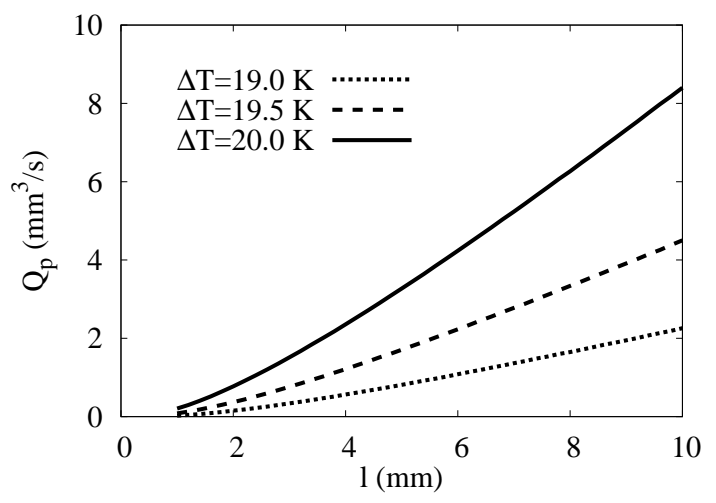

(c) Dependence of $Q_{p}$ on $l$

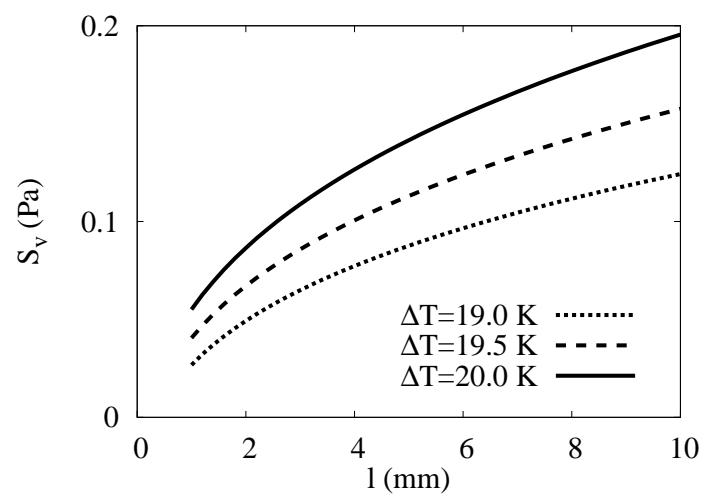

(e) Dependence of $S_{v}$ on $l$

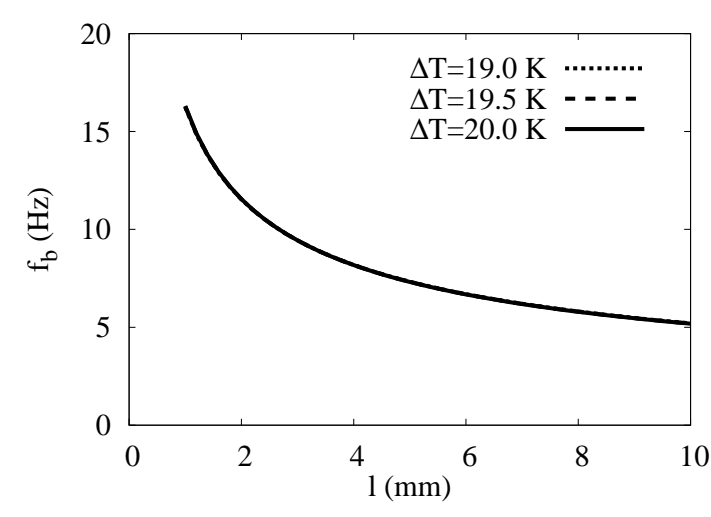

(b) Dependence of $f_{b}$ on $l$

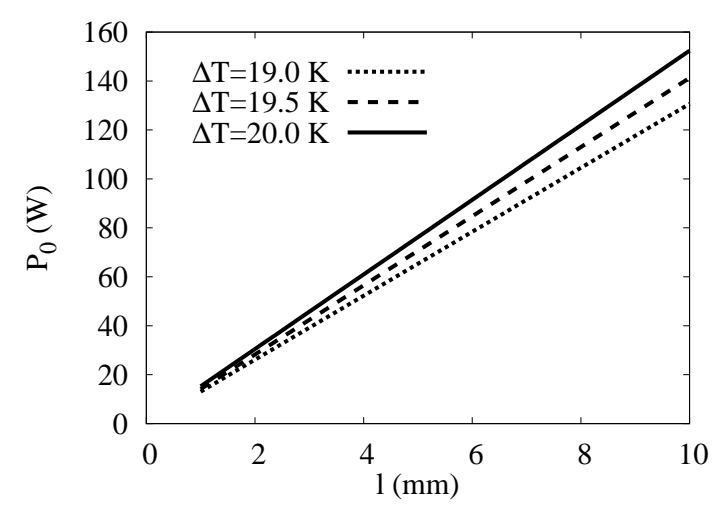

(d) Dependence of $P_{0}$ on $l$

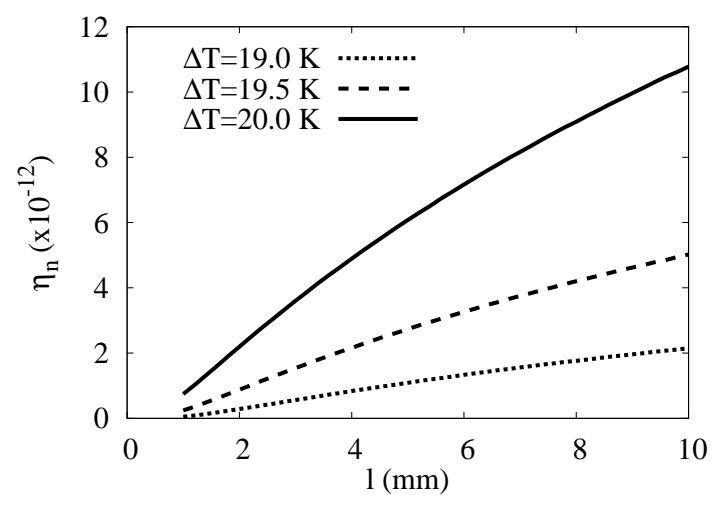

(f) Dependences of $\eta_{n}$ on $l$

FIG. 9. The efficiency for the ASDP. Here, we assume that $d=2 \mathrm{~mm}, w=2 \mathrm{~mm}, l_{1}=l_{2}=l$, and $m_{1}=m_{2}$.

regime. In other words, only the region swept differently in the outgoing and return routes produces a net flow. Note that although a real net volume flow rate (or a net area flow for two dimensional problems) in a channel depends on the channel structure, Khaderi et al. [9] numerically showed that the net area flow is approximately proportional to an area swept, 
which corresponds to $S$, and the proportional constant (efficiency) increases sharply with Reynolds number. In other words, $Q_{p}$ (discussed above) shows the maximum ability for the real net volume flow rate. Thus, we can define the dimensional efficiency $\eta_{d}$ as

$$
\eta_{d} \equiv Q_{p} / P_{0}
$$

However, $\eta_{d}$ is extensive and thus it is not very useful for comparing performance of devices of various sizes. Therefore, we multiply $\eta_{d}$ by the viscous stress $S_{v}$ and obtain the dimensionless efficiency $\eta_{n}$, which has a clear physical meaning (the ratio of power used for driving flow), as

$$
\eta_{n} \equiv Q_{p} S_{v} / P_{0}
$$

where

$$
S_{v}=\frac{12 \mu l\left(\frac{l}{2}\right) \omega_{\max }}{l w}=6 \mu\left(\frac{l}{w}\right) \omega_{\max }
$$

and $l=l_{1}=l_{2}$.

Specifically, Figs. 9(a), 9(b), 9(c), 9(d), 9(e), and 9(f) show the dependences of $S, f_{b}, Q_{p}$, $P_{0}, S_{v}$, and $\eta_{n}$ on $l$, respectively. As $l$ decrease, $S$ decreases rapidly as shown in Fig. 9(a), whereas $f_{b}$ increases moderately as shown in Fig. 9(b). Consequently, as shown in Fig. 9(c), $Q_{p}$ decreases moderately as $l$ decreases. Furthermore, as $l$ increases, $P_{0}$ increases linearly as shown in Fig. 9(d), whereas $S_{v}$ increases gently as shown in Fig. 9(e). Thus, as shown in Fig. 9(f), $\eta_{n}$ decreases monotonically as $l$ decreases.

\section{F. Efficiency of our current device}

We have succeeded in demonstrating the asymmetrical motion of the thermal cilium with an asymmetric joint structure of $\Delta Y_{a} \sim 1.5 \mathrm{~mm}$ at $q=3.3 \mathrm{MW} / \mathrm{m}^{2}$ for $L=19.31 \mathrm{~mm}$. Since the area $S$ of the trajectory in Fig. $3(\mathrm{a})$ is $\sim \frac{1}{2} \Delta Y_{a} \Delta X_{3} \sim 2.1 \mathrm{~mm}^{2}$, a potential net pumping volume flow rate $Q_{p}$ is estimated as $Q_{p} \sim S \phi_{m} f_{b} \sim 2.2 \mathrm{~mm}^{3} / \mathrm{s}$, where $\Delta X_{3} \sim 3 \mathrm{~mm}$ is the width of the maximum trajectory of $\boldsymbol{X}_{3}$ in the $x$ direction, $\Delta Y_{a} \sim 1.4 \mathrm{~mm}$, and $f_{b} \sim 4.5 \mathrm{~Hz}$. Thus, our cilium has a potential ability to realize a pumping function. That is, we obtain $\eta_{d} \sim 2.2 / 107.9=0.02\left(\mathrm{~mm}^{3} / \mathrm{s}\right) / \mathrm{W}$ for our asymmetrical pendulum in Fig. 3 , while $\eta_{d}=0$ for the previous symmetrical pendulum [8]. In other words, we have first demonstrated a useful motion of a thermal artificial cilium that uses a self-propelled phenomenon due to bubbles in a nucleate- to film-boiling regime [8]. Furthermore, we can estimate that $S_{v} \sim$ 
$6 \mu \omega_{\max } L_{h} / 2 \phi_{N i C r} \sim 1.5 \mathrm{~Pa}$ for our experiments, where $\omega_{\max } \sim U_{\max } / L \sim 120 / 19.3 \sim 6.2$ $\mathrm{rad} / \mathrm{s}$. Thus, we obtain $\eta_{n}=\eta_{d} S_{v} \sim 30 \times 10^{-12}$ for our experiments. Moreover, since the theoretical value for the double pendulum in Fig. 9 (f) is approximately $10 \times 10^{-12}$ at $l=10$ $\mathrm{mm}$ and $\Delta T$, we consider that the experimental value is reasonable, although they are not compared directly because of the difference of their conditions.

Hanasoge et al. [4] showed that the magnetic cilia consisting of $n_{e}(\sim 12)$ elements of length $l(=0.2 \mathrm{~mm})$ and width $w(=0.02 \mathrm{~mm})$ produce a net flow of pumping rate of 0.183 $\mathrm{mm}^{3} / \mathrm{s}$. Thus, we obtain $Q_{p} \sim 0.183 / 12 \sim 0.015 \mathrm{~mm}^{3} / \mathrm{s}$ for the single magnetic cilium. Furthermore, if we assume that $f_{b} \sim 24 \mathrm{~Hz}$ [10], we obtain $S_{v} \sim 6 \mu L f_{b} \sim 1.44 \mathrm{~Pa}$. In addition, since Hanasoge et al.'s device is driven by the magnet connected to a DC motor, we can estimate $P_{0} \sim 0.75 \mathrm{~V}$. Thus, we obtain $\eta_{d} \sim 0.015 / 0.75 \sim 0.02\left(\mathrm{~mm}^{3} / \mathrm{s}\right) / \mathrm{W}$ and $\eta_{n} \sim 0.02 \times 1.44 \sim 30 \times 10^{-12}$ for Hanasoge et al.'s device. That is, we find that $\eta_{n}$ of our thermal cilium at $l \simeq 20 \mathrm{~mm}$ is the same level as that of Hanasoge et al.'s device at $l=0.2$ mm. However, $\eta_{n}$ decreases rapidly as $l$ decreases as shown in Fig. 9(f). Thus, we need to improve the efficiency more in the future.

\section{G. The placement of the current work}

One may consider that a surprising aspect of the real natural cilium would be its efficient pumping despite its smallness. In this sense, previous studies of artificial cilia mostly aim at achieving smallness and efficiency at the same time as the ultimate objective, while fabrication of multiple (arrayed) cilia is another focus of interest. However, in our understanding, most of the studies for the artificial cilia have not been in the stage to consider input power as a real problem yet. In other words, most of researchers seem not to have pursued to reduce input power of the artificial cilia to the level of the power corresponding to the efficiency of ATP (adenosine triphosphate) in the natural cilia, as their immediate goal. Therefore, large magnetic equipment that consumes large electric power has been used so far for the magnetic artificial cilia without discussing the input power in detail; e.g., Vilfan et al. [11] used three orthogonal pairs of coils to control their self-assembled magnetic cilia, while Evans et al. [3] used a permanent magnet to drive their magnetic cilia consisting of nanorod arrays. Similarly, although the efficiency of our current device might be insufficient we also aim at achieving smallness and efficiency at the same time as the ultimate objectives. 


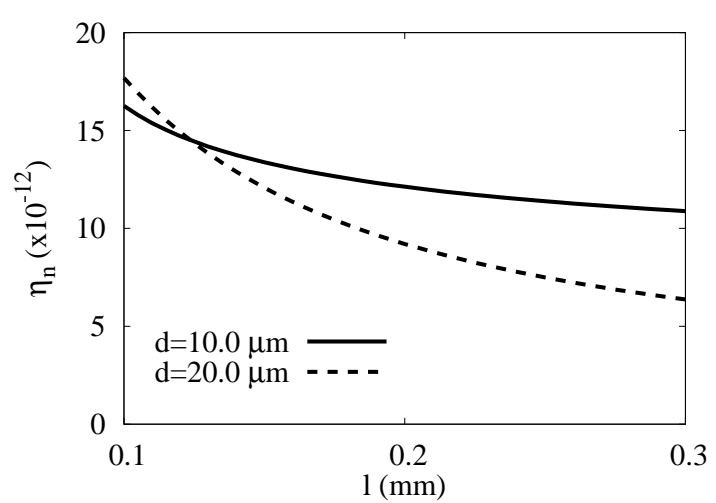

(a) Dependence on $\eta_{n}$ on $l$

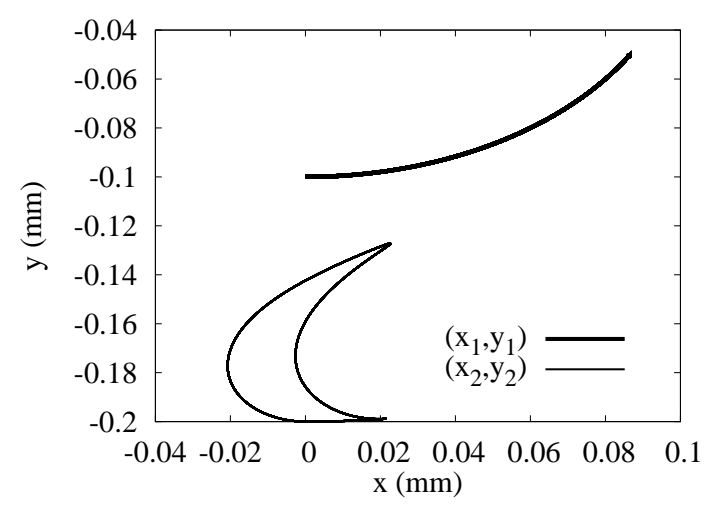

(c) Trajectory of the ASDP

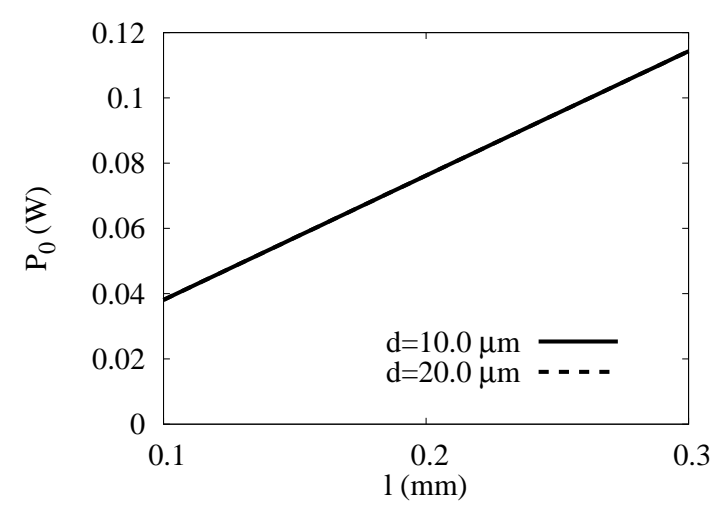

(b) Dependence of $P_{0}$ on $l$

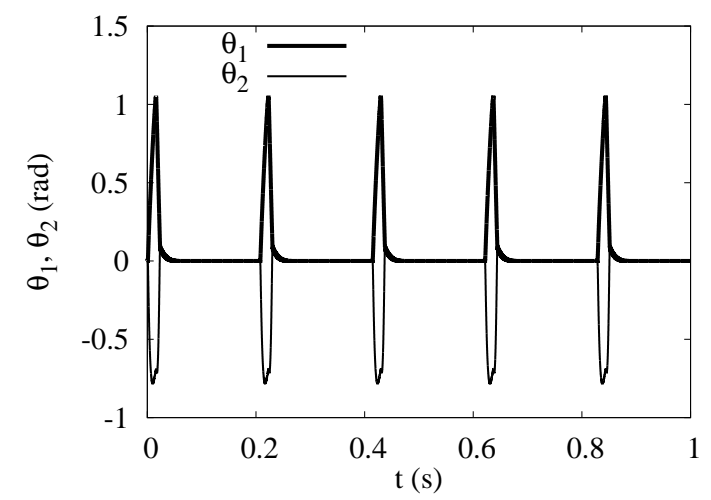

(d) Dependences of $\theta_{1}$ and $\theta_{2}$ on $t$

FIG. 10. The efficiency of the ASDP using thin film beams. Here, $w=50 \mu \mathrm{m}$ and $\Delta T=20.0 \mathrm{~K}$. In (c) and (d), $l=100 \mu \mathrm{m}$ and $d=10 \mu \mathrm{m}$.

Through this study, we have developed a micro heat engine that works in water with a simple structure consisting of a heater and a pair of bend wires, although we demonstrated a self-propelled swing motion of the pendulum whose beam is partially immersed in water [8]. By using this new micro heat engine, we have first succeeded in demonstrating a high-speed beating motion of a thermally-driven artificial cilium in a nucleate- to film-boiling regime. Surprisingly, the maximum velocity of the tip of the cilium is approximately $12 \mathrm{~cm} / \mathrm{s}$ at $q \geq 2.8 \mathrm{MW} / \mathrm{m}^{2}$. Therefore, we consider that our thermal artificial cilium is useful. In addition, we have a chance to achieve smallness and efficiency at the same time in the future by considering an optimal design. For example, Figs. 10(a) and 10(b) show the dependences of $\eta_{n}$ and $P_{0}$ on $l$, respectively, under the condition that $\Delta T=20 \mathrm{~K}, w=50 \mu \mathrm{m}$, and $d=10$ to $20 \mu \mathrm{m}$, while Figs. 10(c) and 10(d) show the trajectory and the time evolution (of $\theta_{1}$ and $\left.\theta_{2}\right)$, respectively at $l=100 \mu \mathrm{m}$ and $d=10 \mu \mathrm{m}$. That is, by using thin film beams, we have 
a chance to achieve $\eta_{n} \sim 10^{-11}$ and $P_{0} \sim 0.1 \mathrm{~W}$ at $l \sim 200 \mu \mathrm{m}$ in the future, although the detail analysis is a beyond the scope of this manuscript. Note that since the moment of inertia of a rod is larger than that of a film, the motion (amplitude) of the film is larger than the rod. Thus, $S$ of the film becomes larger than that of the rod. This is the intuitive explanation about why films are more efficient than rods.

\section{CONCLUSION}

In conclusion, we have first reported that an asymmetrical joint structure connected to the micro heat engine consisting of a heater and a pair of bend wires shows an asymmetric beating motion with high speeds of the order of $5 \mathrm{~cm} / \mathrm{s}$ at $q \geq 2.3 \mathrm{MW} / \mathrm{m}^{2}$. In particular, by using an asymmetric joint structure having asymmetric flexibility between the forward and recovery motions, we have demonstrated the asymmetrical motion of the thermal cilium.

[1] C. Eloy and E. Lauga, Phys. Rev. Lett. 109, 038101 (2012).

[2] J. den Toonder and P. Onck, Trends Biotechnol. 31, 85 (2013).

[3] B. A. Evans, A. R. Shields, R. L. Carroll, S. Washburn, M. R. Falvo, and R. Superfine, Nano Letters 7, 1428 (2007).

[4] S. Hanasoge, P. J. Hesketh, and A. Alexeev, Microsystems \& Nanoengineering 4, 11 (2018).

[5] J. d. Toonder, F. Bos, D. Broer, L. Filippini, M. Gillies, J. de Goede, T. Mol, M. Reijme, W. Talen, H. Wilderbeek, V. Khatavkar, and P. Anderson, Lab Chip 8, 533 (2008).

[6] T. Masuda, A. M. Akimoto, K. Nagase, T. Okano, and R. Yoshida, Science Advances 2 (2016).

[7] H. Sugioka, Phys. Rev. Applied 3, 064001 (2015).

[8] H. Sugioka and M. Kubota, Journal of the Physical Society of Japan 89, 064402 (2020).

[9] S. N. Khaderi, M. G. H. M. Baltussen, P. D. Anderson, J. M. J. den Toonder, and P. R. Onck, Phys. Rev. E 82, 027302 (2010).

[10] S. Hanasoge, M. Ballard, P. J. Hesketh, and A. Alexeev, Lab Chip 17, 3138 (2017).

[11] M. Vilfan, A. Potočnik, B. Kavčič, N. Osterman, I. Poberaj, A. Vilfan, and D. Babič, Proceedings of the National Academy of Sciences 107, 1844 (2010). 